\title{
A fuzzy comprehensive evaluation and entropy weight decision-making based method for power network structure assessment
}

\author{
Yuguo Qi ${ }^{1}$, Fushuan Wen ${ }^{2}$, Ke Wang ${ }^{3}$, Li Li ${ }^{3}$, S.N.Singh ${ }^{4}$ \\ (1. School of Electrical Engineering, South China University of Technology, Guangzhou 510640, CHINA; \\ 2. School of Electrical Engineering, Zhejiang University, Hangzhou 310027, CHINA; \\ 3. Guangdong Power Dispatching Center, Guangzhou 510640, CHINA \\ 4. Department of Electrical Engineering, Indian Institute of Technology Kanpur, INDIA)
}

\begin{abstract}
With the rapid development of the Chinese electric power industry, the working load of staff members in some provincial power dispatching centers has increased tremendously and may cause potential threats to the secure operation of power systems. The working load can be reduced by transferring the dispatching rights of some equipment and power lines to the lower-level local dispatching centers, but the security of the power system will a major concern. The suitability of the dispatching right devolution for some parts of a power system depends mainly on the network structure and criticality of the system. In this work, a few indices are first developed to reflect the power network structure, and then a new method for the comprehensive assessment of the power network structure is presented through the combined use of the fuzzy comprehensive evaluation and the entropy weight decision-making method. The fuzzy set approach is employed to identify the membership degree of each index to various evaluation results, whereas the entropy weight method is used to acquire the sets of weighting factors. This method represents a unified one of the quantitative results obtained from the power structure data and the qualitative results based on the judgment of domain experts. Finally, practical examples are served for demonstrating the developed approach.
\end{abstract}

Key words: Dispatching right, power network structure, fuzzy comprehensive evaluation, entropy weight decision-making method, assessment index

\section{Introduction}

The electric power grid in China is expanding continuously, leading to an increased working load of the dispatchers in many folds and bringing new challenges to the electrical network dispatching department. Yi et al. (2007) and Sun et al. (2005) have discussed the shifting of power grid operating rights, and proposed the principle and specific implementation methods of the operating rights devolution. In the long run, the dispatching rights devolution, rather than the operating right devolution, is the most effective way of reducing the dispatchers' working load and of raising the efficiency. The dispatching rights devolution has to respect certain constraints on the power grid structure and it is not possible to carry out the devolution process in every power grid structure. Therefore, before the dispatching right devolution, it is important and necessary that the dispatching rights devolution adaptability to each power grid structure is appraised.

For evaluating the dispatching rights devolution adaptability to the power grid structure, this paper proposes a power grid structure indicator system using the synthetic evaluation by the fuzzy set theory and entropy weight method. The proposed indicator system in this work not only weighs each power grid structure's overall suitability regarding the dispatching right devolution, but also reflects the microscopic transitional condition of each power grid structure. 


\section{The evaluation index system of the power grid structure}

The proposed indicator system represents an evaluating indicator set in which all indicators are chosen according to the appraisal system of the pre-specified goal (Su et al., 2005). The development of the evaluation system needs to observe technicality, rationality and feasibility. The implemented "Guide on Security and Stability for Power Systems" and "The Power Transmission Network Security Appraisal (Implementation)" have been referred to screen and optimize each target, including one first-level target, six second-level targets and thirteen third-level targets.

"The fitness of the power grid structure to the dispatching rights devolution" is taken as the first-level target, which is judged based on the degree of the dispatching rights devolution. Six second-level targets including clarity, jurisdiction, flexibility, reliability, security and economy have been used for the comprehensive and systematic evaluation of the power grid structure. Regarding each second-level target, the segmentation has been proposed by thirteen third-level targets. These indicators are constituted for the power grid structure’s comprehensive evaluation index system as shown in Fig. 1.

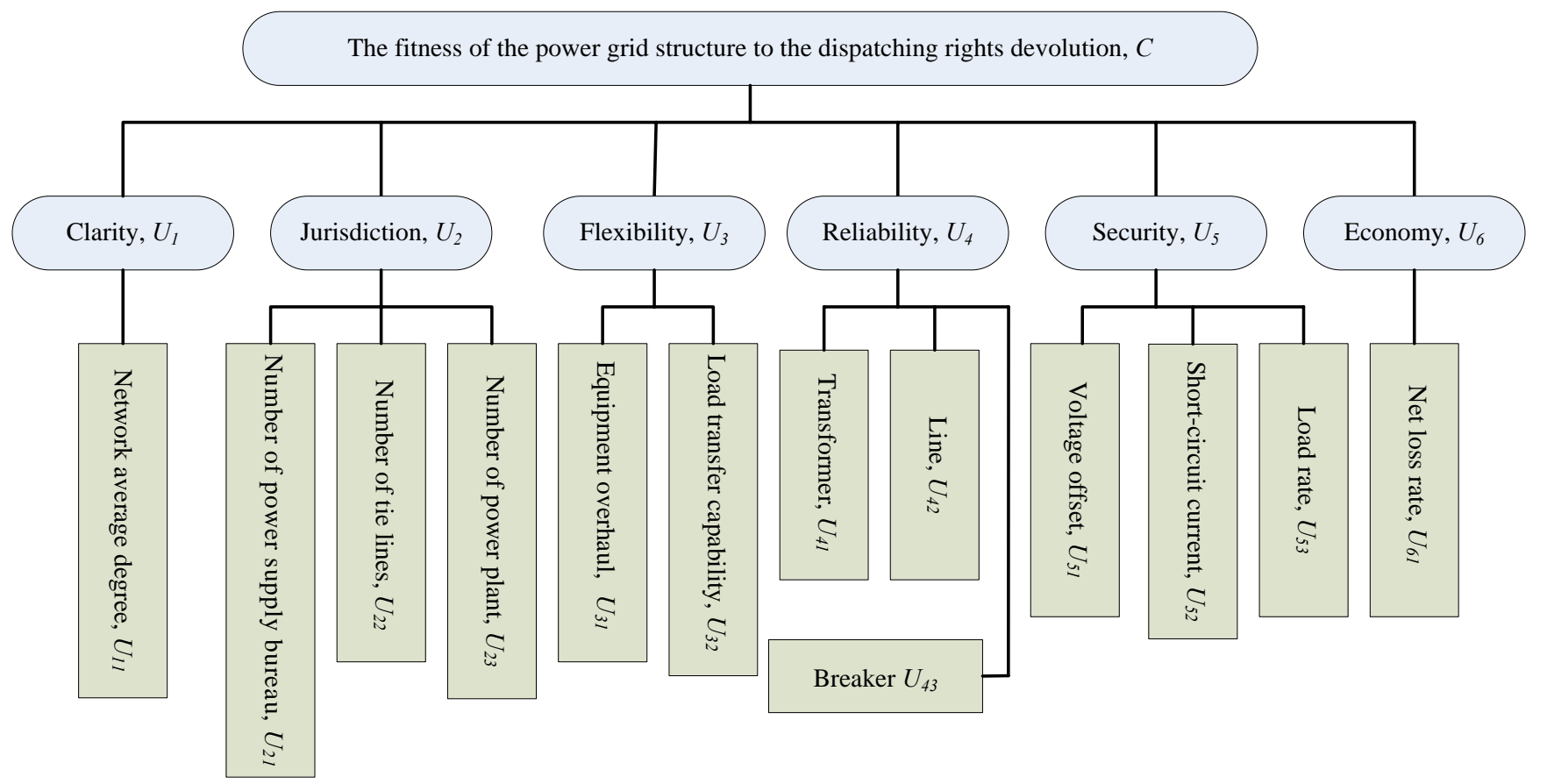

Fig. 1 Indices for assessing the power grid structure

\section{The entropy method and fuzzy comprehensive evaluation}

The entropy method (Lin, et al., 2009) is an object empowerment approach, in which the weight values of individual indicators are determined by calculating the entropy and entropy weight. The greater the entropy is, the smaller the corresponding entropy weight will be. The amount of useful information that the target provides to the decision-maker is reduced. If the entropy weight is zero, it provides no useful information to the decision-maker, and this indicator may be removed (Jing et al., 2009). The main steps of the entropy weight method include: the formation of the evaluation matrix; the standardization of the evaluation matrix; the calculation of the entropy and the entropy weight.

The fuzzy comprehensive evaluation is also known as the fuzzy synthetic decision-making (Li et al, 2006). The key to the method is to determine the weight set. The weight can be determined by several methods, e.g., the subjective method using the analytic hierarchy process (Zhao et al., 2004), the objective method such as the comprehensive exponential method (Guo et al, 2009). The entropy weight method can establish a quantifiable bridge between the subjective and objective methods, and represents a good way to determine weights. Thus, the comprehensive evaluation method for determining the evaluation matrix and the entropy weight method for determining the weights of indicators so as to build the grid structure of synthetic evaluation model will be used in this work. The main steps of the developed method are as follows:

a) Determine the factor set and evaluation set ;

b) Establish the fuzzy evaluation matrix ; 
c) Determine the weight of each index layer using the entropy weight method ;

d) Calculate the compound decision vector.

\section{A model for evaluating the grid structure}

4.1 Formation of the fuzzy evaluation matrix: For the fuzzy evaluation matrix $\boldsymbol{R}=\left[r_{i j}\right](i=1,2, \ldots, m ; j=1,2, \ldots, n)$, whose elements $r_{i j} \in[0,1]$ are the membership degree of the $i$ th index to the $j$ th reviewer. Therefore, the evaluation set should first be determined before the formation of the assessment matrix.

4.1.1 Establishment of the evaluation set: The evaluation set is an index appraisal criterion. The evaluation set is usually divided into five levels, namely outstanding, good, medium, bad, and very bad. The corresponding five evaluation levels values are $1,0.8$, $0.6,0.4,0.2$. The evaluation set $\boldsymbol{V}$ is formed as $\boldsymbol{V}=\left[V_{1}, V_{2}, \ldots, V_{5}\right]$, and $V_{i}(\mathrm{i}=1,2, \ldots, 5)$ represents the $i$ th evaluation level.

4.1.2 Determination of the membership degree: The qualitative and quantitative indicators' membership degrees are determined separately, as detailed below.

1) Determination of the membership degree for qualitative indicators. The experts' scores are used to determine the membership degree such as the kind of qualitative indicators "Equipment overhaul”, "Load transfer capability". For example, for indicator "Equipment overhaul” , assuming that the total number of experts is $n$, with $m_{i}(\mathrm{i}=1,2, \ldots, 5)$ experts believe that the structure of this grid belongs to the evaluation level $V_{i}(i=1,2, \ldots, 5), \sum_{i=1}^{5} m_{i}=n$, then $r_{i}=m_{i} / n$ is the indicator "Equipment overhaul” belonging to various levels of the membership evaluation. In this way, the indicator of the single-factor evaluation matrix can be expressed as follow:

$$
\left[m_{1} / n, m_{2} / n, m_{3} / n, m_{4} / n, m_{5} / n\right]
$$

2) Determination of the membership degree for quantitative indicators: For the indicators shown in Fig. 1, most of them are quantitative ones. Only the indicator "Available factor of lines" belongs to the benefit-type one, whereas the other indicators are the loss-type ones. For the treatment of the quantitative indicators, membership functions must be formed first. There are a variety of ways for the formation of functions. The semi-trapezoidal and trapezoidal distributions (Qin, 2003) are employed to determine the membership functions.

For the loss-type indicators, such as "Network average degree", "Number of tie lines", generally the membership function can employ the following form:

$$
\underset{\sim}{A}(x)=\left\{\begin{array}{cc}
1 & x<a \\
\frac{b-x}{b-a} & a \leq x \leq b \\
0 & x>b
\end{array}\right.
$$

For the benefit-type indicators, such as "Available factor of lines”, generally the membership function can utilize the following form:

$$
\underset{\sim}{A}(x)=\left\{\begin{array}{cc}
0 & x<a \\
\frac{x-a}{b-a} & a \leq x \leq b \\
1 & x>b
\end{array}\right.
$$

where $a, b$ are constants.

When the membership functions are formed, the membership degrees of the indicators to the evaluation level $V_{i}$ can be obtained by putting the measured value of indicators into the membership function. It should be noted that there are many ways to determine the membership function of fuzzy sets, but usually with a certain amount of subjectivity.

Combining experts' scores, the values of "Network average degree" corresponding to the evaluation set of outstanding, good, medium, bad, very bad are specified as 1.5, 2, 2.5, 3, 4. Then according to the method described above, the membership functions can be obtained, as shown in Eqns. (4)-(8).

For example, if "Network average degree" of a grid structure is 1.86, then substituting this value into Eqns. (4)-(8), the membership degree vector [0.28, $0.72,0,0,0]$ that belongs to the evaluation set $\boldsymbol{V}$ can be obtained. 


$$
\begin{aligned}
& V_{1}(x)=\left\{\begin{array}{cc}
1 & 0<x<1.5 \\
\frac{2-x}{2-1.5} & 1.5 \leq x<2 \\
0 & x \geq 2
\end{array}\right. \\
& V_{2}(x)=\left\{\begin{array}{cc}
\frac{x-1.5}{2-1.5} & 1.5 \leq x<2 \\
\frac{2.5-x}{2.5-2} & 2 \leq x<2.5 \\
0 & \text { otherwise }
\end{array}\right. \\
& V_{3}(x)=\left\{\begin{array}{cc}
\frac{x-2}{2.5-2} & 2 \leq x<2.5 \\
\frac{3-x}{3-2.5} & 2.5 \leq x<3
\end{array}\right. \\
& V_{4}(x)=\left\{\begin{array}{cc}
0 & \text { otherwise } \\
\frac{4-x}{4-3} & 3 \leq x<4 \\
0 & \text { otherwise }
\end{array}\right. \\
& V_{5}(x)=\left\{\begin{array}{cc}
\frac{x-2.5}{3-2.5} & 2.5 \leq x<3 \\
\frac{x-3}{4-3} & 3 \leq x<4 \\
0 & \text { otherwise }
\end{array}\right.
\end{aligned}
$$

4.1.3Formation of the fuzzy evaluation matrix (Wang, et al., 2009): As shown in Fig.1, the first-level index $U_{t}$ contains $k$ subordinate targets, where $U_{t}$ can be either the top-level general indicator or one of the second-level indicators. Then $U_{t}$ can be divided into the following $k$-factor set: $U_{t}=\left\{U_{t l}, U_{t 2}, \ldots, U_{t k}\right\}$. Here, the single-factor evaluation matrix of the $i$ th sub-set is as follows:

$$
\boldsymbol{U}_{\boldsymbol{t i}}=\left[u_{t i 1}, u_{t i 2}, u_{t i 3}, u_{t i 4}, u_{t i 5}\right] \quad i=1,2, \ldots, k
$$

where $u_{t i j}(i=1,2, \ldots, k ; j=1,2, \ldots, 5)$ is the membership degree of the $i$ th index of $U_{t}$ relative to the $j$ th evaluation level.

Thus, the evaluation matrix of $U_{t}$ can be obtained as following after forming every single-factor evaluation matrix.

$$
\boldsymbol{R}_{t k \times 5}=\left|\begin{array}{cccc}
u_{t 11} & u_{t 12} & \ldots & u_{t 15} \\
u_{t 21} & u_{t 22} & \ldots & u_{t 25} \\
\ldots & \ldots & \ldots & \ldots \\
u_{t k 1} & u_{t k 2} & \ldots & u_{t k 5}
\end{array}\right|
$$

From the index system shown in Fig. 1, seven evaluation matrices can be formed. The meanings of the evaluation matrices are listed in Table 1.

4.2 Determining the weight by the entropy weight method: When the evaluation matrix is obtained, the next step is to determine weights. Because the elements of the evaluation matrix formed by the fuzzy set theory $r_{i j} \in[0,1]$, there is no need to standardize the evaluation matrix. The entropy weight value and weights can be obtained directly by calculating the evaluation matrix.

For the evaluation matrix $\boldsymbol{R}=\left[r_{i j}\right]_{m \times n}$, the entropy of the $i$ th indicator is defined as follow:

$$
\boldsymbol{H}_{\boldsymbol{i}}=-k \sum_{j=1}^{n} r_{i j} \ln r_{i j} \quad i=1,2, \cdots, m
$$


Table 1 The fuzzy evaluation matrix (FEM)

\begin{tabular}{|c|c|c|c|}
\hline NO. & Name & Order & Meaning \\
\hline 1 & $\boldsymbol{R}_{\boldsymbol{u} 1}$ & $1 \times 5$ & FEM of clarity \\
\hline 2 & $\boldsymbol{R}_{\boldsymbol{u} 2}$ & $3 \times 5$ & FEM of jurisdiction \\
\hline 3 & $\boldsymbol{R}_{\boldsymbol{u} 3}$ & $2 \times 5$ & FEM of flexibility \\
\hline 4 & $\boldsymbol{R}_{\boldsymbol{u} 4}$ & $3 \times 5$ & FEM of reliability \\
\hline 5 & $\boldsymbol{R}_{\boldsymbol{u} 5}$ & $3 \times 5$ & FEM of security \\
\hline 6 & $\boldsymbol{R}_{\boldsymbol{u} \boldsymbol{}}$ & $1 \times 5$ & FEM of economy \\
\hline 7 & $\boldsymbol{R}_{\boldsymbol{c}}$ & $6 \times 5$ & Comprehensive evaluation matrix \\
\hline
\end{tabular}

where, $k=1 / \ln n$.

The entropy weight of the $i$ th indicator is defined as follows:

$$
\omega_{i}=\frac{1-H_{i}}{m-\sum_{l=1}^{m} H_{l}}
$$

As can be seen from the above, $0 \leq \omega_{i} \leq 1$ and $\sum_{i=1}^{m} \omega_{i}=1$

The entropy weight value is taken as the weight of each indicator. There is no need to calculate the entropy weight value if there is only one third-level indicator, such as the clarity and economy, because the entropy weight value is 1 for them.

4.3 The Comprehensive Evaluation: After the evaluation matrix $R=\left[r_{i j}\right]_{m n}$ and the weight vector $\omega_{1 \times m}$ are obtained, the comprehensive evaluation results of the current level can be determined through fuzzy arithmetic operations, then the final evaluation results can then be obtained through the stepwise computation, as formulated below:

$$
\boldsymbol{B}=\omega_{1 \times k} \circ R_{k \times 5}
$$

where, $\omega_{1 \times k}$ is the weight vector corresponding to the current-level indicator; $R_{k \times 5}$ is the evaluation matrix corresponding to the current-level indicator; “॰” is the fuzzy operator. Different operators can be used for solving different practical problems. The model $\mathrm{M}(\wedge, \vee)(\mathrm{Qin}, 2003)$ is employed here, in which the element $b_{j}$ of $\boldsymbol{B}$ is as,

$$
b_{j}=\underset{h=1}{\stackrel{k}{\vee}}\left(\omega_{h} \wedge r_{h j}\right)
$$

The final comprehensive evaluation matrix can be obtained by calculating from the third-level indicators layer by layer in Fig. 1. According to the maximum membership degree law (Ramot, et al, 2002), the comprehensive evaluation level of the grid structure is the largest element of the matrix corresponding to the comprehensive evaluation level.

The final score of the grid structure can also be obtained by the evaluation grade scores mentioned above and the evaluation results vector calculated by Eqn. (13). The evaluation grade scores could be written in a vector form as,

$$
\boldsymbol{F}=[1,0.8,0.6,0.4,0.2]
$$

The evaluation result vector $\boldsymbol{B}$ obtained by Eqn. (13) needs to be normalized and could then be denoted as $\boldsymbol{B}^{\prime}$. The final score of the power grid structure is obtained as

$$
T=\boldsymbol{B}^{\prime} \boldsymbol{F}^{T}
$$

\section{Case studies}

A numerical example is used to demonstrate the developed comprehensive evaluation method. The simulation data of the grid structure are listed in Table 2.

Table 2 The values of power grid structure indices

\begin{tabular}{|c|c|c|c|c|c|c|c|c|c|c|c|}
\hline Indices & $U_{11}$ & $U_{21}$ & $U_{22}$ & $U_{23}$ & $U_{41}$ & $U_{42}$ & $U_{43}$ & $U_{51}$ & $U_{52}$ & $U_{53}$ & $\boldsymbol{U}_{61}$ \\
\hline Values & $\mathbf{2 . 4 1 1 8}$ & $\mathbf{4}$ & $\mathbf{7}$ & $\mathbf{8}$ & $\mathbf{1 . 8 8 4}$ & $\mathbf{9 9 . 3 0 5}$ & $\mathbf{9 . 0 2 3}$ & $\mathbf{1 . 2 4}$ & $\mathbf{4 7 . 6}$ & $\mathbf{3 . 6 0}$ & 4.3 \\
\hline
\end{tabular}

Based on the experts' experience and various references, the evaluation vectors of each third-level indicator can be computed 
using Eqns. (1)-(10) with the membership function for quantitative indicators and scores of experts for qualitative indicators. The simulation results are presented in Table 3.

Table 3 The evaluation vector of the third-level indices

\begin{tabular}{|c|c|}
\hline Indices & Evaluation vector \\
\hline$U_{11}$ & {$[0,0.1764,0.8236,0,0]$} \\
\hline$U_{21}$ & {$[0,0,0.1562,0.7344,0.0058]$} \\
\hline$U_{22}$ & {$[0.1023,0.4951,0.0670,0,0]$} \\
\hline$U_{23}$ & {$[0,0.2030,0.5401,0.0105,0]$} \\
\hline$U_{31}$ & {$[0.3333,0.3333,0.3333,0,0]$} \\
\hline$U_{32}$ & {$[0.1111,0.2222,0.3333,0.3333,0]$} \\
\hline$U_{41}$ & {$[0.301,0.203,0.110,0,0]$} \\
\hline$U_{42}$ & {$[0.105,0.256,0.408,0,0]$} \\
\hline$U_{43}$ & {$[0,0,0.104,0.356,0.4056]$} \\
\hline$U_{51}$ & {$[0.101,0.243,0.289,0.106,0]$} \\
\hline$U_{52}$ & {$[0.465,0.236,0.109,0,0.012]$} \\
\hline$U_{53}$ & {$[0.460,0.230,0.020,0.014,0]$} \\
\hline$U_{61}$ & {$[0.203,0.368,0.109,0,0]$} \\
\hline
\end{tabular}

According to Eqn. (10), $U_{l 1}$ in Table 3 forms the second-level evaluation matrix of clarity $R_{l}$. Then the second-level evaluation matrices i.e. $R_{2}, R_{3}, R_{4}, R_{5}, R_{6}$ can be obtained by the same way, corresponding to the second-level indicators of jurisdiction, flexibility, reliability, security, and economy. Each of the second-level evaluation matrices is as follows:

$$
\begin{aligned}
& R_{1}=\left[\begin{array}{lllll}
0 & 0.1764 & 0.8236 & 0 & 0
\end{array}\right] \quad R_{2}=\left[\begin{array}{ccccc}
0 & 0 & 0.1562 & 0.7344 & 0.0058 \\
0.1023 & 0.4951 & 0.0670 & 0 & 0 \\
0 & 0.2030 & 0.5401 & 0.0105 & 0
\end{array}\right] \\
& R_{3}=\left[\begin{array}{ccccc}
0.3333 & 0.3333 & 0.3333 & 0 & 0 \\
0.1111 & 0.2222 & 0.3333 & 0.3333 & 0
\end{array}\right] \quad R_{4}=\left[\begin{array}{ccccc}
0.301 & 0.203 & 0.110 & 0 & 0 \\
0.105 & 0.256 & 0.408 & 0 & 0 \\
0 & 0 & 0.104 & 0.356 & 0.4056
\end{array}\right] \\
& R_{5}=\left[\begin{array}{ccccc}
0.101 & 0.243 & 0.289 & 0.106 & 0 \\
0.465 & 0.236 & 0.109 & 0 & 0.012 \\
0.460 & 0.230 & 0.020 & 0.014 & 0
\end{array}\right] \quad R_{6}=\left[\begin{array}{ccccc}
0.203 & 0.368 & 0.109 & 0 & 0
\end{array}\right]
\end{aligned}
$$

By using $R_{1}, R_{2}, R_{3}, R_{4}, R_{5}, R_{6}$ the entropy weight vectors $\omega_{1}, \omega_{2}, \omega_{3}, \omega_{4}, \omega_{5}, \omega_{6}$ are computed using Eqns. (11)-(12).The result is given in Table 4. Then, the evaluation vectors of the second-level indicators can be obtained according to Eqn. (13), and is shown in Table 5. The final evaluation matrix $R$ can be obtained from Table 5. The final entropy weight vector $\boldsymbol{\omega}$ and the final evaluation vector $\boldsymbol{B}$ can be calculated using the same method. The final result is

The final score obtained by Eqn. (15) is

$$
\boldsymbol{B}=[0.2,0.24,0.44,0.11,0.01]
$$

$$
T=\boldsymbol{B F}^{T}=0.7020
$$

Table 4 The weighted vectors of $R_{1}, R_{2}, R_{3}, R_{4}, R_{5}, R_{6}$

\begin{tabular}{|c|c|}
\hline Indices & The entropy weight \\
\hline clarity & $\omega_{1}=1$ \\
\hline jurisdiction & $\omega_{2}=[0.3775,0.3196,0.3029]$ \\
\hline flexibility & $\omega_{3}=[0.6136,0.3864]$ \\
\hline reliability & $\omega_{4}=[0.3926,0.3261,0.2814]$ \\
\hline security & $\omega_{5}=[0.2717,0.3163,0.4119]$ \\
\hline economy & $\omega_{6}=1$ \\
\hline
\end{tabular}


From Table 5 and in accordance with the maximum membership degree law, it is found that the clarity of this grid structure is medium; jurisdiction is poor; reliability is medium; security is outstanding; economy is good. However, from the perspective of jurisdiction, this grid structure needs to be improved. This grid structure is found to be good from the final score, and the devolution of the dispatching rights of this grid structure can be taken into account.

Table 5 The evaluation vector of the second-level indices

\begin{tabular}{|c|c|c|}
\hline Indices & Vector form & The evaluation vector \\
\hline clarity & $\boldsymbol{\omega}_{\mathbf{1}} \circ \boldsymbol{R}_{\boldsymbol{1}}$ & {$[0,0.1764,0.8236,0,0]$} \\
\hline jurisdiction & $\boldsymbol{\omega}_{\mathbf{2}} \circ \boldsymbol{R}_{\mathbf{2}}$ & {$[0.1023,0.3196,0.3029,0.3775,0.0058]$} \\
\hline flexibility & $\boldsymbol{\omega}_{\mathbf{3}} \circ \boldsymbol{R}_{\mathbf{3}}$ & {$[0.3333,0.3333,0.3333,0.3333,0]$} \\
\hline reliability & $\boldsymbol{\omega}_{\mathbf{4}} \circ \boldsymbol{R}_{\mathbf{4}}$ & {$[0.301,0.203,0.3261,0.2814,0.2814]$} \\
\hline security & $\boldsymbol{\omega}_{\mathbf{5}} \circ \boldsymbol{R}_{\mathbf{5}}$ & {$[0.4119,0.243,0.2717,0.106,0.012]$} \\
\hline economy & $\boldsymbol{\omega}_{\mathbf{6}} \circ \boldsymbol{R}_{\mathbf{6}}$ & {$[0.203,0.368,0.109,0,0]$} \\
\hline
\end{tabular}

\section{Conclusions}

This paper presents an evaluation index system for the power grid structure, and develops the power grid structure evaluation method based on the fuzzy comprehensive evaluation and entropy weight method. This proposed method is a good combination of subjectivity and objectivity, and provides both qualitative and quantitative analysis. Through simulations and practical applications, the index system and the evaluation method presented in this paper have been proven to be reasonable and effective.

\section{Acknowledgement}

This work is supported by the Key Project in the National Science \& Technology Pillar Program with project number 2008BAA13B10.

\section{References}

Guo S.H., Lin Z.J., Hong X.Q. and She R.X., 2009. On the application of composite index method based on entropy authority to the water quality evaluation. Environmental Science and Management, Vol.34, No.12, pp.165-167.

Jing L.P. and Michael K. N., 2009. An entropy weighting k-means algorithm for subspace clustering of high-dimensional sparse data. IEEE Transactions on Knowledge and Data Engineering, Vol.19, No.8, pp.1026-1041.

Li Y.H. and Hu Y.Q., 2006. A model of multilevel fuzzy comprehensive evaluation for investment risk of high and new technology project. proceeding of 2006 International Conference on Machine Learning and Cybernetics, China pp.1942-1947.

Lin Z.Z. and Wen F.S., 2009. Entropy weight based decision-making theory and its application to black-start decision-making. Proceedings of the CSU EPSA, Vol.21, No.6, pp.26-33.

Qin S.K., 2003. Principle and application of comprehensive evaluation. $1^{\text {st }}$ ed. Electronic Industry Press, Beijing China, 227p.

Ramot D., Milo R. and Friedman M., 2002. Complex fuzzy sets. IEEE Transactions on Fuzzy Systems, Vol.10, No.2, pp.171-186.

Su X. and Tang W., 2005. Index system of rural electric network performance assessment. Journal of Shenyang Agricultural University, Vol.36, No.3, pp.721-723.

Sun J.H., Zhang M.L. and Li D.P., 2005. Discussion on adjustment of the electric power dispatch relationship in new situation. Proceedings of the 29th China Electric Power Dispatching and Operation, China.

Wang Q., Wen F.S., Liu M. and Yi S., 2009. Combined use of fuzzy set theory and analytic hierarchy process for comprehensive assessment of electricity markets, Automation of Electric Power Systems, Vol.33, No.7, pp.32-37.

Yi S.M., Li S., Chen H.K. and Lin S.H., 2007. Discussion on change in operating mode of Guangdong power grid dispatching. Guangdong Electric Power, Vol.20, No.3, pp.1-6.

Zhao Y.F. and Chen J.F., 2004. Analytic hierarchy process and its application in power system. Electric Power Automation Equipment, Vol.24, No.9, pp.85-88. 


\section{Biographical notes}

Yuguo Qi received his BE degree from Zhengzhou University, China in 2008, and is currently a master student in South China University of Technology, China. His main research interest is power system analysis.

Fushuan Wen received his BE and ME degrees from Tianjin University, China, in 1985 and 1988, respectively, and PhD from Zhejiang University, China, in 1991, all in electrical engineering. He joined the faculty of Zhejiang University in 1991, and has been a full professor and the director of the Institute of Power Economics and Information since 1997. He had been a university distinguished professor, the deputy dean of the School of Electrical Engineering and the director of the Institute of Power Economics and Electricity Markets in South China University of Technology (SCUT), China from 2005-2009. His current research interests lie in power industry restructuring, power system alarm processing, fault diagnosis and restoration strategies, as well as smart grids.

Ke Wang is a senior engineer in Guangdong Power Dispatching Center. He received his BE and ME degrees from North China Electricity University, China, both in electrical engineering. His main research interest lies in power system dispatching mechanism.

$\mathbf{L i ~ L i ~ i s ~ a ~ s e n i o r ~ e n g i n e e r ~ i n ~ G u a n g d o n g ~ P o w e r ~ D i s p a t c h i n g ~ C e n t e r . ~ H e r ~ m a i n ~ r e s e a r c h ~ i n t e r e s t ~ l i e s ~ i n ~ p o w e r ~ s y s t e m ~ d i s p a t c h i n g ~ m e c h a n i s m . ~}$

S.N. Singh received his M. Tech. and Ph.D. from Indian Institute of Technology Kanpur, India in 1989 and 1995, respectively. Presently, he is working as Professor in the Department of Electrical Engineering, Indian Institute of Technology Kanpur, India. His research interests include power system restructuring, power system optimization \& control, voltage security and stability analysis, power system planning, and ANN application to power system problems. He is a Fellow of IE (India), Fellow of IETE (India), member of IET (UK) and senior member of IEEE.

Received November 2009

Accepted March 2010

Final acceptance in revised form June 2010 